\title{
Research on Applications of Image Processing Technologies in Intelligent Transportation
}

\author{
Tinghui Ye \\ (ZheJiang Police College, Hangzhou City,Zhejiang Province, 310051)
}

\begin{abstract}
Keywords: Image processing technologies, Intelligent transportation, Smart transportation
\end{abstract}
\begin{abstract}
At present, image processing technology has been widely used in intelligent transportation management in our country. It has greatly improved the traffic supervision and control, the technical support for the stable traffic operation and the improvement of the level of urbanization. This paper analyzes the common image processing technologies, including the image gray transformation technology, the image binarization technology and the image denoising technology. It gives the application models of image processing technologies in intelligent transportation, to provide some references for the relative researchers
\end{abstract}

\section{Introduction}

Intelligent transportation system is to solve the problems caused by increased vehicles and traffic chaos situation, which can improve the traffic operation efficiency and improve the safety level of traffic intelligent management system for the operation. At present, it has been widely used in many countries all over the world, and has become the main management means of the transportation management department. Intelligent transportation system is mainly used in traffic information service system, public transportation system, electronic toll collection system and emergency management system. Intelligent transportation system is the modern means of information technology, electronic technology, digital processing technology and intelligent technology combined, all-round, multi angle of supervision and management for traffic, improve traffic management in China's road safety and stability. At the same time, the intelligent transportation systems can also reduce the traffic management departments in our country, the human material and financial resources. It is necessary for China's economic and social development. Intelligent transportation system, and to develop based on the construction of road design based on the system, the main application of the advanced technology of various information technology, intelligent technology, electronic technology, image processing technology, sensor technology and so different. Among them, the image processing technology is the most widely used and most important in the intelligent transportation system. With the application of these advanced technologies, intelligent transportation system has become an advanced, accurate and real-time traffic system, which can drive traffic and transportation enterprises to develop in an intelligent way. Image processing technology is the most widely used technology in the intelligent transportation system. Image processing technology has become the most important technical means of intelligent transportation system in our country.

\section{Common Image Processing Technologies}

Image Gray Transformation Technology. Image gray transformation refers to the method of changing the gray values of each pixel in a source image according to certain transformation conditions according to some target condition. The purpose is to improve the image quality, so that the image display effect is clearer. The image gray level transformation processing is a very basic and direct spatial domain image processing method in the image enhancement processing technology, and is an important component of the image digitization software and the image display software. The general imaging system only has a certain range of luminance response, and the ratio of the maximum to the minimum is called the contrast. Because of the limitation of the imaging system, the disadvantage of the contrast is often insufficient, which makes the visual effect of the human eye very 
poor. Gray transformation refers to the method of changing the gray values of each pixel in a source image according to certain transformation conditions according to some target condition. The purpose is to improve the image quality, so that the image display effect is clearer. The image gray level transformation processing is a very basic and direct spatial domain image processing method in the image enhancement processing technology, and is an important component of the image digitization software and the image display software. The gray level transform function should be based on the nature of the image and the purpose of processing. An image containing a license plate taken by a camera or camera is usually a bitmap of color, but there are also some 24-bit true color bitmaps. Before you do image processing, you need to convert 24-bit true colors into 256 grayscale images. Each pixel of 24-bit true color image is represented by 3 bytes, corresponding to R, G, B. The converted black and white image is still expressed in bytes. Three components of R, G, B are equal value. The relation between them is:

$$
\operatorname{Gray}(\mathrm{i}, \mathrm{j})=0.299 * \mathrm{R}(\mathrm{i}, \mathrm{j})+0.588 * \mathrm{G}(\mathrm{i}, \mathrm{j})+0.114
$$

In the above formulation, Gray( $(i, j)$ is the gray level of the $(i, j)$ point after the gray transformation.

Image Binarization Technology. The binarization processing of the image is that change the gray value of the point on the image into 0 or 255. The whole image shows a clear black and white effect. The gray image is obtained by the appropriate threshold value, and the binarization image, which can still reflect the whole and local features of the image, is obtained. In the digital image processing, the binarization image occupies a very important position. This is because, on the one hand, some processing such as text image and fingerprint image of engineering drawings, etc. the image itself is another aspect of the binarizations, in some cases even if the image itself is gray, we also try to make it into binarization image and the gray image processing, namely the value of three. This is because considering the practical image processing system, the processing speed, low cost, large amount of information, such as color image processing costs too much. In addition, binarization images can be analyzed and described in terms of geometry, which is much better than gray scale images. If the image before preprocessing is binarization, this step can be omitted. In the license plate recognition system in actual image of binarization transformation is the key to determine the appropriate threshold value, the character and background can be separated, and the binarization transform the image must have good conformality, not to lose shape information, no extra vacancy. At the same time, the license plate recognition system requires fast processing and large amount of information. Processing with binarization images can greatly improve the processing efficiency. The key to binarizations is to find appropriate values to distinguish objects from backgrounds. Let the original gray level image be $f(x, y)$, and the binarization image is $g(x, y)$. The process of binarizations is expressed as follows:

Among the formulation, $\mathrm{t}=[\mathrm{f}(\mathrm{x}, \mathrm{y}),(\mathrm{x}, \mathrm{y}), \mathrm{N}(\mathrm{x}, \mathrm{y})]$.

$$
\mathrm{g}(\mathrm{x}, \mathrm{y})=\left\{\begin{array}{l}
1, f(x, y) \geq t \\
0, f(x, y)<t
\end{array}\right.
$$

Image Denoising Technology. Image denoising is the process of reducing noise in digital images, called image denoising. In the process of digitization and transmission, realistic digital images are often affected by imaging equipment and external environment noise, which are called noisy images or noisy images. Noise is an important cause of image interference. In practical applications, there may be a variety of noise, which may occur during transmission or in quantization. One of the main tasks of image preprocessing is smoothing images to remove noise. Noise elimination methods can be divided into spatial or frequency domain, which can be divided into global processing or local processing. They can also be distinguished by linear filtering, nonlinear filtering and adaptive filtering. The mean filter based on neighborhood averaging method is very suitable for removing the particle noise in the image obtained by scanning. The domain average method effectively suppresses the noise, and at the same time leads to fuzzy phenomena due to averaging. The degree of fuzziness is proportional to the radius of the field. The smoothness of the geometric mean filter can be compared to the arithmetic average filter, but less detail is lost in the filtering process. The inverse harmonic mean filter is more suitable to deal with impulse noise, but it has a drawback is the need to know the noise is dark noise or light noise, to facilitate the selection of the filter order right symbol, if the order 
of the symbol the wrong choice may cause disastrous consequences. Set the function of an image is $\mathrm{f}$ $(\mathrm{x}, \mathrm{y})$, and the filtered image is $\mathrm{g}(\mathrm{x}, \mathrm{y})$. The filtered image is obtained by the following formulation:

$$
\mathrm{g}(\mathrm{x}, \mathrm{y})=\frac{1}{m} \sum_{s \in(i, j)} f(i, j)
$$

The algorithm is simple and fast. But its drawback of the algorithm is to reduce noise while blurring the image. At edges and details, the larger the neighborhood is, the worse the blur is.

\section{Applications of Image Processing Technologies in Intelligent Transportation}

Detection of Mobile Vehicles. The main interference factors that affect the correct detection of moving vehicles in the road traffic monitoring system are vehicle shadow, projection of night head lights, mutual occlusion between vehicles and the vibration of camera. These interference factors are often encountered in the actual application environment and cannot be avoided. Therefore, a practical traffic monitoring method must consider how to eliminate these disturbances. In the choice of block size, block size selected and the actual monitoring scene and monitoring related to the size of the block basically meet the requirements of each block are almost a single movement or no motion is not too small, otherwise there will be matching based on gray level contains the same pattern on the block, to you can search to ensure the correct block. The disadvantage of block matching is that it cannot solve the occlusion problem. If there is a large area of occlusion in the object movement, the calculation error will occur. The aberration method is a basic method of motion analysis of image sequences, which relies on the detection of the significant differences between two adjacent frames to detect moving objects. The difference method of video sequence is applied to detect the human or larger target in the image sequence, if there is movement. The method is easy to implement with small amount of computation and is easy to implement, and the actual effect is better. Therefore, it is also the preferred method of real-time traffic monitoring system, and it is also the focus of our discussion. In the differential image method, there are two kinds, one is the difference between the current frame and the background frame, and the other is the difference between adjacent frames. The human visual process is selective. For the same visual stimulus, the conclusions obtained by different people may be different, and the results of observation are also related to the prior knowledge of the observer, and are very affected by experience. The learning process is the accumulation process of experience, and the guidance of experience plays an important role in the production of the result. When the matching between observation and a priori knowledge reaches a certain level, it will produce a reaction to perception.

Extraction of Mobile Vehicles. To distinguish the moving vehicle from each image with independent connected region from the difference image space and extract the features of each moving object, it is necessary to judge the topological connectivity of the image. In this paper, the sequential region growing method is used to identify the topological connectivity of the region in the process of image search, and the moving region is in the gray space of the moving image. The basic concept of order region growing method is to find a seed point in the image, from the point to the neighborhood search, and has the same neighborhood gray feature points are placed in a region, and the region number to sign, until no new point can be put into this area. Then, find new seed points and start new areas of growth. Moving object tracking is a complex process. It first needs to initialize the new moving target, obtain the initialization parameters of the moving object, and search the best matching of the initialized moving target in the next frame. More complex is, because the vehicle is moving during the phenomenon of occlusion, so in the analysis process, but also the moving target merger and decomposition and many other considerations. This section will present a method suitable for moving vehicle tracking. The end of the growth after all pixels have been assigned to an area. The moving vehicle obtained by the differential detection method has the vehicle shadow besides the body itself. Because of this, the vehicle shadow cannot be separated from the vehicle itself, so it brings a lot of negative effects to the recognition and tracking, which leads to a great improvement in error rate and a decrease in the overall performance of the system. Therefore, the vehicle shadow must be eliminated so that the result of vehicle outer rectangle detection is closer to the true value. 
Because of the presence of light, there is a shadow around the car. Shadows come from all or part of the object. Shadow detection methods have many, such as in space using brightness, chroma, saturation three aspects of information, establish model for shadow detection and recognition. The algorithm holds that the shaded points in the background have the same color as before the cover, but the brightness is low. Therefore, the shadow mask is used for shadow detection of the points where the gradation of the image to be detected is obviously changed.

Tracking of Mobile Vehicles. Monitoring and tracking technology in time for operation of the vehicle, tracking and real-time analysis of moving objects, real-time analysis and control of specific operating conditions on the road conditions and individual vehicles, to ensure smooth and safe operation of the road. The sequence of motion target tracking technology in the military, national defense, traffic monitoring, industrial process control, medical research, aircraft navigation, traffic statistics, astronomical observation and other areas has a very important practical value and broad prospects for development. The purpose of moving object tracking is to analyze the sequence image and calculate the displacement of moving object in the continuous frame image, and give the moving parameters such as moving target speed and so on. Capturing and tracking motion objects of interest to us using images. In complex background, moving target tracking is used to achieve specific purpose. Reliability and accuracy are two important indexes in tracking process. Moving object tracking is a complex problem, and the key is the matching of objects. First, a set of features belonging to a single moving object is defined, such as scale, shape, intensity, etc., and the features of these regions are very different from those of adjacent regions. Region tracking is a good way to overcome occlusion problems because these features provide sufficient information for tracking. Region tracking is especially suitable for tracking in static background, because when we extract moving objects from static background, these moving objects are often very different from those of static objects around them. Due to the moving target as a point to find matching is too simplified, easy to cause many errors in judgment, so this paper will use rectangle motion region to express, this is equivalent to the external rectangle matching tracking, the target symbol, make the judgment more accurate, more credible results. For vehicles serial initialization, used here is the nearest neighbor method, this method is convenient and needs less information is known, because of the emergence of new target to estimate the motion parameters of it, so according to the proximity in space on it and characteristics of objects and large differences in small degree. Currently, most region tracking methods rely on previous detection to distinguish moving targets and then track targets. It relies on the dynamic system in the two influence each other, this system is captured in the scene is projected to the shape and motion changes with time in the image plane, using the filter tracking technology accurate estimation of regional geometry and velocity.

\section{Conclusion}

With the continuous improvement of computer technologies, image processing technology has been widely used in the management of intelligent transportation in our country. The relevant administrative departments should actively learn from the advanced management experience at home and abroad, fully consider the actual situation of the operation of China's road, and deepen the image processing technologies in intelligent transportation system to improve the efficiency of traffic management in our country.

\section{References}

[1] Hu Zhuping. Research and Application of the Intelligent Video Searching of Facial Images in Public Transportation [J]. Software Industry and Engineering, 2015(5): 52-56.

[2] Sun Qiang. On the Application of Digital Image Processing Technology to Smart Traffic [J]. Journal of Jilin Engineering Normal University, 2016, 32(7): 92-94. 
[3] Jin Lin, Lin Yuhong, Guo Jiangang. Study on Inspection System of Timber Transportation Based on Image Recognition and Mobil Communication [J]. Journal of Transportation Engineering and Information, 2012, 10(4): 13-18.

[4] Wei Xiaowei. The use of video image processing technology in the intelligent transportation system [J]. Electronic Test, 2015(6): 106-108. 